\title{
Subject Index Vol. 58, 1996
}

Acoustic neuroma 32, 224, 277

- $\quad$ rhinometry 157

Adaptation 301

Aerodynamics 326

Agenesis, sphenoid sinus 347

Allergic rhinitis 157

Amikacin 68

$\alpha$-Amino-3-hydroxy-5-methyl-4-isoxazole-

propionic acid 121 Anti-obstructive effect 157 Aspirin 61 Atopy 82 ATP 320

Audiological examination 233 Auditory brainstem response(s) 61,219,

224

ossicle 143

pathway 262 Aviation 304 Azelastine nasal spray 157

Bacterial interference 147

Benign paroxysmal positioning vertigo 277

Bilateral hearing loss 175

Breathiness 326

Caloric testing 42 cAMP-activated channels 183 Canal-wall-down technique 39 Carcinoma 167

Cartilaginous incision 51 Cauterization 271 Cell junction 280 Cepstrum 326 Cerebrospinal fluid

164 c-Fos 262 Children 78 Cholesteatoma 23, 39 Chronic sinusitis 311 Clinical correlation 315

Cochlea 1 Cochlear hair cells 1

implant 127,298

nucleus, dorsal, ventral 262 Cochleovestibular nerve 295 Cockayne's syndrome 343 Cockpit crew 304

Colony formation 248 Complement 151 Computed tomography 298, 333 Computer-assisted surgery 46 Congenital deformity 333 Continuous interleaved sampling 127

Cultured human mucosal microvascular

endothelial cells 320 Cyst 311

Dacryocystorhinostomy 311 Damped pendular rotation test 204 Damped-rotation test 343

Deafness 333 Decompression 46 [18F]Deoxyglucose 195 Dissection 271 Dysphagia 105

Dysphonia 326

Ear surgery 39

Electron microscopy 213

Electronystagmography 42

Electrophysiology 121

ELISA 175

Endocrine orbitopathy 46

Endolymphatic hydrops 271,338

sac 271 Endonasal surgery 87 Endothelin-1 4 Enteral nutrition 253 Eosinophil 82 
granule protein 82 Epidermis 280 Equilibrium test 233 Esophagus 105

External auditory canal 136, 280 Extranodal lymphoma 171

Facial movement 32

- $\quad$ nerve lesion 13, 266

Fascia temporalis 36

Fibrin glue 27

Fibroblast growth factors 248

Fibrous dysplasia 55

'Finger-like projections' 136, 280

Fistula 333

Focal metabolic activation 195

Fura-2 320

Fusobacterium necrophorum 178

Gentamicin, intratympanic 277

Glutathione 68

Guinea pig(s) 4,27,68, 121,271

Harada's disease 233 Head and neck 167

- $\quad$ - - cancer 253

Head-tilted position 204

Healthy young females 110

Hearing impairment 219

Hemidesmosome 280

High-resolution computed tomography 200

Histology 27

Horizontal nystagmus 204

Human temporal bone 208

Hypoglossal-facial nerve anastomosis 32

Immunocytochemistry 262 Immunoglobulins 151 Immunohistochemical staining 82

- $\quad$ study 288

Immunohistochemistry 23, 121 Infection 151

Inferior colliculus 262 Inner ear 333

- melanocytes 233

hair cells 121

Intracellular calcium concentration 320 Intracerebral haemorrhage 164 Iron deposits, deep cervical lymph nodes 164

JunB 262

Keratinocytes $23 \mathrm{Ki}-6723$

Lacrimal sac 311 Laryngeal lymphoma 171

- neoplasm 171

Laryngoscopy 326

Laser-assisted uvulopalatoplasty 99, 243 Leiomyosarcoma 115 Lemmierre's syndrome 178 Light microscopy 136 Local anaesthetics 306

- $\quad$ anesthesia 99

Low-vacuum scanning electron microscope

9 Lymphatic drainage, humans 164

Macrophage 288 
Magnetic resonance imaging 295, 315, 333

Malignant lymphoma 171

Maxillary sinus neoplasms 93

Mechanoreceptors 1

Meningitis 333

Ménière's disease 304

N-Methyl-D-aspartate 121

Middle-ear surgery 143

Morphology 136,213

353

Motor cortex 266 Mouse 136,280 Mucosal immunity 151 Multicellular spheroids 248

Multifrequency tympanometry 78 Multipeak speech coding 127 Mumps 295, 338 Myofascial pain 306 Myringoplasty 27

Nasal cavity 115

mucosa 229,320

polyp(s) 82,229

sinuses 151 Nasolacrimal duct 311 Nasopharynx 147 Neurofilament 13

Neuronal loss, vestibular nuclei 343 Neuropeptides 229 Neuroplasticity 13 Non-Hodgkin's

lymphoma 171 Normal flora 147

- population 315

Nucleolar organizer region 93

Nystagmus, spontaneous 42

Ocular counterrolling 301

Olfaction 183

Organophosphorus compound intoxication

219 Orthostatic dysregulation 110 Otitis media with effusion 78,147 Otoacoustic emissions 61

Otoconia 9 Otolithic membrane 9 Otosclerosis 143 Ototoxicity 68 Outer hair cells 121

Pain 99

Papillary carcinoma 238

Paranasal sinus 115,347

Pathology 68

Perceptual rating 326

Percutaneous endoscopic gastrostomy 253

Phonation flow 326

Phosphorylation 13

Pilots 304

Politef implant 143

Positron emission tomography 195

Primary auditory cortex 195

Prognosis 93, 167

Prolonged tilt 301

Quantitative analysis 200

Radical mastoidectomy 39 Rat 262

Regeneration 13 Repetitive stimulation 262 Revision tympanoplasty 36 Rhinomanometry 157

Roughness 326 Rubella 74

Saccule 4 
Saddle nose 51

Salsave ${ }^{\circledR} 258$

Salty taste recognition threshold 258

Scanning electron microscopy 136

Schellong test 110

Sea water 87

Semicircular canal 4

Sensorineural hearing loss 74

Septoplasty 51

Sinus 55

Sinusitis 315

Sleep apnea 99

Slow-phase eye velocity 204

Snoring 99

Soft tissue sarcoma 115

Spantide 121

Spectral-peak speech coding 127

Speech coding 127

- understanding 127

Stapedial otosclerosis 200

Stimulus rate 224

$\alpha$-Streptococcus 147

Subrenal capsule assay 248

Substance P 121

Sudden deafness 295, 338

Surgery 115

Surgical anatomy 208

- $\quad$ approach 243

Symptom score 157

Synaptic reorganization 266

Systolic pressure decrease 110

T lymphocyte 288 Taste 258

- dysfunction 258

Temporal bone 298

Temporomandibular joint dysfunction 306 Three-dimensional imaging 298 Thrombosis 178

Thyroglossal cyst 238

Thyroid 238

Timing of anastomosis 32

Tinnitus patients 195

Tip links 1

Tonsillitis 178

Tracheobronchomalacia 288

Transcription factors 262

Transduction 183

Transmission electron microscopy 280

Tumor cell-fibroblast interaction 248 
Tumour-associated tissue eosinophilia 167

Turbinate 115

Tympanic membrane 136, 280

- $\quad$ - perforation 27

Ultrasound 105 Utricle 4 Uvulopalatopharyngoplasty 99

Vertigo 277, 295

Vestibular ganglion, human 213

hair cells 1

membranous labyrinth 208

nerve 208 Vestibulo-ocular reflex 301 Viral infection 175 Visual input 301

Voice acoustics 326

- pathology 326

Washing, nasal cavities 87

354

Subject Index Vol. 58, 1996 